\title{
Hydrodynamic education with rheoscopic fluid
}

\author{
Daniel Duda ${ }^{1, *}$, Marek Klimko ${ }^{1}$, Radek Škach $^{1,2}$, Jan Uher $^{1,2}$, and Václav Uruba ${ }^{1,3, * *}$ \\ ${ }^{1}$ Department of Power System Engineering, University of West Bohemia in Pilsen, Univerzitní 22, 30614 Pilsen, Czech Republic \\ ${ }^{2}$ Doosan Škoda Power, Tylova 57, 30128 Pilsen, Czech Republic \\ ${ }^{3}$ Institute of Thermomechanics, Czech Academy of Sciences, Dolejškova 5, 18200 Prague, Czech Republic
}

\begin{abstract}
We present a educational poster supporting the subject „Mechanics of fluids I“, which the students evaluate to be difficult mainly due to abstractness. Our goal is to show in vivo the behavior, especially the non-linearity, of various flows transiting into turbulence. The fluid motion is visualized by using the rheoscopic fluid, which consist of water and the dust of mica, whose particles are longitudinal and shiny resulting into easily observable reflections, when the particles coherently orient along the maximum stress. This happens mainly in shear layers, e.g. at the boundary between vortex core and envelope. An example of flow transiting into turbulence is the Taylor-Couette flow between two concentric cylinders, which with increasing Taylor number passes through various regimes from fully laminar bearing flow through the Taylor vortex flow (TVF) and later Wavy vortex flow (WVF) up to Turbulent Taylor vortices regime (TTV) and, finally, the regime of featureless turbulence.
\end{abstract}

\section{Motivation}

The flowing media as water and air are important part of nature and therefore hydrodynamics is an important part of the engineering education for the entire existence of the human civilization, which has been established in order to build huge irrigation systems in the plains of southern Iraq in $4^{\text {th }}$ millennium B.C. [1].

Hydrodynamics has, similarly as any other physical discipline, two main aspects: it is practical and it is gorgeous. Of course, it is also difficult. So difficult that many scientists declare, the turbulence is the last unsolved problem of classical mechanics [2]. The last can be seen as an advantage for the student, because then the curse ,Mechanics of fluids I" contains only some of the easily solvable basic problems, but still, the students do not like this subject as can be seen from Table 1 showing the number of students of the curse and their final grades and Figure 1 showing the number of obtained points in the final test. Table 1 shows that the pass-ability is quite good, but the grades not - students are able to learn the minimum needed, but the subject did not interested them. Similarly, Figure 1 with distribution opposite than the Gaussian one shows, that there are lot of students able to solve the problems in the test very well, probably they could solve even more difficult problems, but on the other hand, there is lot of students working just for the needed minimum.

We all (the authors and You, dear reader) know that hydrodynamics is fascinating. But students do not share this opinion with us. The question is, how to show it to them.

\footnotetext{
*e-mail: dudad@kke.zcu.cz

**e-mail: uruba@kke.zcu.cz
}

Table 1. The number of students of the curse „Mechanics of fluids I“ in the academic year 2017/2018.

\begin{tabular}{l|c|c}
\hline Students which & {$[\#]$} & {$[\%]$} \\
\hline registered for the curse & 145 & 100.0 \\
passed the test & 122 & 84.1 \\
failed the test & 11 & 7.6 \\
did not try & 12 & 8.3 \\
\hline registered for the exam & 122 & 100.0 \\
passed the exam & 122 & 100.0 \\
with grade 1 & 8 & 6.6 \\
with grade 2 & 47 & 38.5 \\
with grade 3 & 67 & 54.9 \\
\hline
\end{tabular}

\section{Rheoscopic flow visualization}

The effect of rheoscopic ${ }^{1}$ or kaliroscopic ${ }^{2}$ behavior is caused by small anisotropic and shiny particles, which are able to coherently orient in some volume bigger than single particle and thus reflect light. Roughly speaking, the particles orient according to the local shear stress in the liquid, but the exact relation to the carrying flow is very complex and not yet fully understood [4].

\footnotetext{
${ }^{1} \rho^{c} \varepsilon \iota v$ - to flow, $\sigma \kappa о \pi \varepsilon \iota v-$ to observe
}

${ }^{2} \kappa \alpha \lambda \circ \varsigma$ - beautiful 


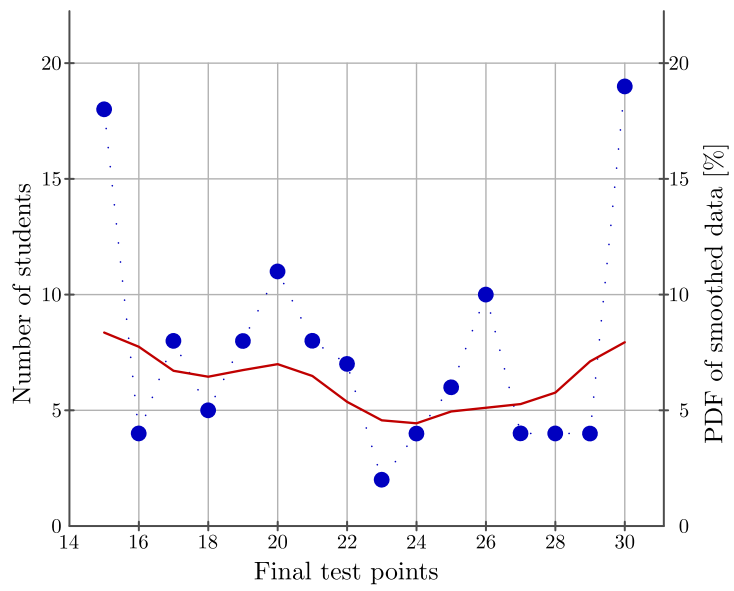

Fig. 1. blue points show the number of students passing the final test with specific number of points; minimum needed for passing was 15 , maximum possible was 30 . The red line shows the probability density function of those numbers smoothed by using the Kolmogorov-Zurbenko filter [3] over 3 neighbors and with 3 iterations.

\subsection{Scientific experiments}

Although the exact interpretation of an image obtained by this technique is nearly impossible, various scientific experiments has been performed by using this method, mainly focusing on some qualitative aspect of the studied flow.

For example, Carlson et al. [5] studied plane Poiseuille flow observing the turbulent spots in otherwise laminar flow. The turbulent spot is easily recognizable as an area of sharped light and dark wild structures. They tracked the turbulent spots evaluating their speed and rate of grow.

It seems that very popular is to use the rheoscopic visualization technique for study of Taylor-Couette flow (see [6] or section 3.1 for more details), which is natural if we consider that both, the flow and the technique, are quite easy to realize not needing large circulation facility or expensive instrumentation and analysis software, which is the case of the crucial work of Andereck, Liu and Swinney [7].

One of the moderner studies was performed by Richter et al. [8], who visualized the Taylor-Couette flow in Taylor-vortex reactor used for mixing in chemical industry. They compared the „standard“ geometry of smooth inner rotating cylinder with a ribbed rotor, i.e. cylinder with superposed plates perpendicular to the rotation axis and placed equidistantly in a such way, that there could be 2 Taylor vortices between them. They found that the ribs immobilize Taylor vortices resulting into shifting the onset to turbulence to higher Taylor numbers. The micromixing is higher, while at the same time the macromixing is reduced.

Berrich et al. [9] also used rheoscopic flow visualization in their study of Taylor-Couette flow with an imposed axial flow. By using an image processing they highlighted individual vortices on the photographs and thus automatized the data analysis.

\subsection{Mica}

As mentioned above, the effect of rheoscopicity is achieved by anisotropic shiny particles. Such particles are massively produced for cosmetics industry and are made of mica ${ }^{3}$. Hence it is easy to buy them, but a disadvantage is, that there is no datasheet available, therefore we do not know basic properties as density, size distribution, asymmetricity, surface roughness etc., which would be needed for use in a proper hydrodynamic experiment. Additionally, the particles are covered by some fancy color of unknown properties and it seems that differently colored particles have different coagulation dynamics.

Figure 2 shows a typical image observable by using mica as a rheoscopic medium. The scene is illuminated by single point-source (Sun), which is more advantageous for seeing clear image than a diffuse light. Wilkinson [4] suggests the use of 3 point light sources of different colors (red, green and blue), which then allows us to distinguish the direction of particle orientation according to the reflected color hue and the degree of particle alignment according to the reflection magnitude.

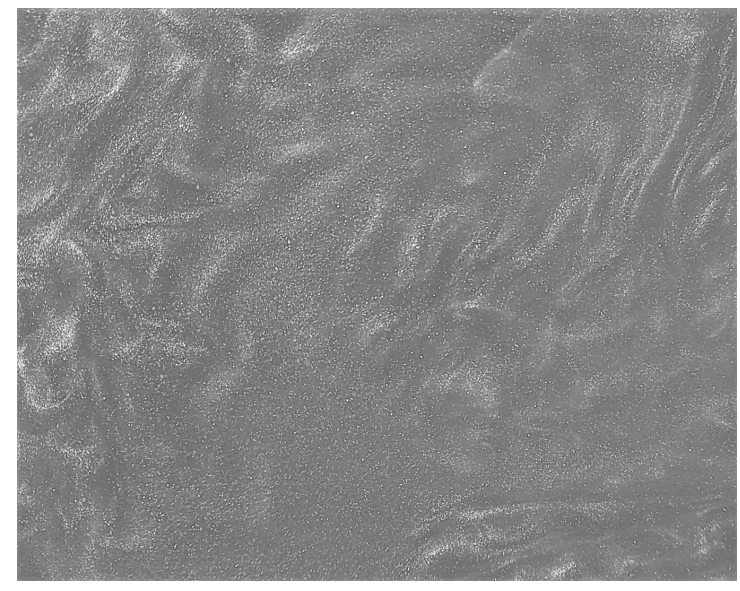

Fig. 2. Photograph of the water + mica solution. Illumination by direct sunlight. Flow is the decayed turbulence in a $6 \mathrm{~mm}$ deep pool covered by plexiglass. The length of the field of view is $8 \mathrm{~cm}$.

\section{Planed apparatus for Taylor-Couette flow}

Inspired by the variety of real scientific experiments performed by using rheoscopic fluids and studying some aspects of the Taylor-Couette flow, we chose this kind of flow as well.

\subsection{Taylor-Couette flow}

Taylor-Couette flow is flow between two concentric cylinders, which rotate (both or only one of them). Therefore the flow is closed and quite easy to perform. Despite its simplicity in boundary conditions, this flow shows lot of

\footnotetext{
${ }^{3}$ Mica = slída
} 
the wealth of hydrodynamics. Instabilities based on inertia, the rise of self-ordered structures, their instabilities and rise of turbulence... hence this flow plays in hydrodynamics similar role as hydrogen atom in chemical physics or Drosophila in biology [6].

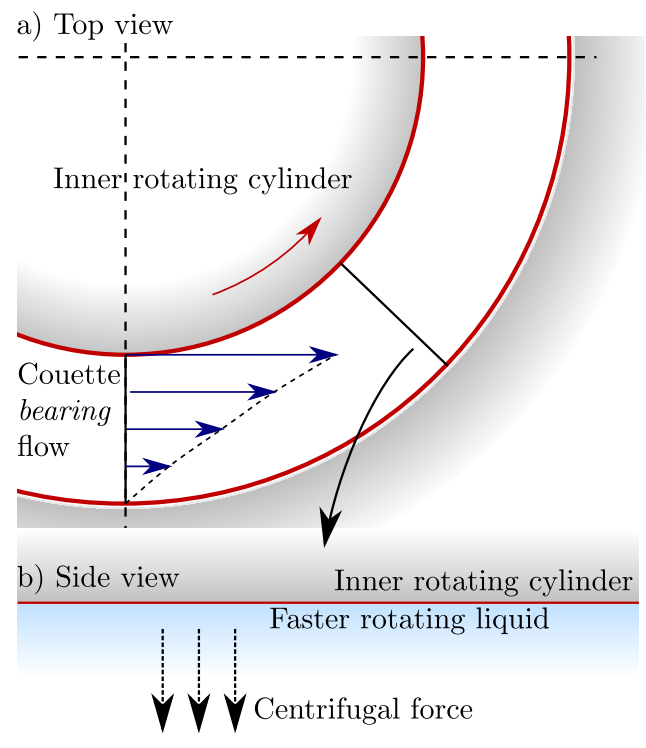

Outer cylinder

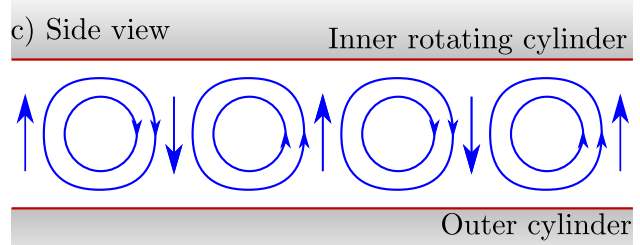

Fig. 3. Sketch of the Taylor instability in the laminar Couette (bearing) flow. Inner cylinder rotates, outer one does not. (a) the top view with depicted base laminar Couette flow; (b) the side view to the cut, the faster rotating fluid at the inner cylinder experiences centrifugal force stronger than the slowly rotating fluid; linear stability of the laminar flow is lost, the new stable flow topology (c) is established consisting of annular vortices with alternate orientation. The flow is still laminar, however the process of transition to turbulence is initiated.

The scenario starts from laminar fully azimuthal flow called Couette or bearing, where the tangential velocity $v_{\vartheta}(r)$ depends on the distance $r$ from rotation axis as [6]

$$
v_{\vartheta}(r)=A r+\frac{B}{r},
$$

where

$$
\begin{aligned}
& A=\frac{\omega_{o}-\omega_{i}\left(\frac{R_{i}}{R_{o}}\right)^{2}}{1-\left(\frac{R_{i}}{R_{o}}\right)^{2}} \\
& B=R_{i}^{2} \frac{\omega_{i}-\omega_{o}}{1-\left(\frac{R_{i}}{R_{o}}\right)^{2}},
\end{aligned}
$$

where $\omega_{i}$ and $\omega_{o}$ are the inner and outer rotation rates, $R_{i}$ and $R_{o}$ are radii of the inner and outer cylinder.
When the Rayleigh circulation criterion of the form

$$
\frac{\mathrm{d}(r v)^{2}}{\mathrm{~d} r}<0
$$

is fulfilled, then the situation is unstable. This instability is called Görtler instability and occurs in flow at convexly curved boundary [10], which is the case of Taylor-Couette flow with outer cylinder standing, or rotating more slowly than the inner one.

Lets look at special case with outer cylinder standing, $\omega_{o}=0$. Then the fluid close to the inner boundary is faster than the fluid at outer one, therefore the inner one experiences stronger centrifugal force pushing it to the outer boundary. Due to the continuity, the fluid at outer boundary is pushed out to the inner boundary; the result is a still laminar system of regular convection-like cells [11], see figure 3 .

This situation is qualitatively similar to the TaylorRayleigh instability, which is originally the instability of heavier fluid placed on top of the lighter fluid in a gravity; its „continuous“ variant is called Rayleigh-Bénard instability and occurs when fluid is heated from bellow in gravity. In the Taylor-Couette case, the inertia (centrifugal force) plays the role of gravity and the higher velocity at moving inner boundary plays the role of lower density caused by heating. (Or it can be seen oppositely, depending what you declare to be more basic).

When the fluid motion is damped via viscosity, then the just described scenario realizes when the Taylor number

$$
T a=\sqrt{\Lambda} R e_{i}=\sqrt{\frac{d}{R_{i}}} \frac{\omega_{i} R_{i} d}{v}=\frac{\omega_{i} d^{3 / 2} R_{i}^{1 / 2}}{v} \geq 41.2
$$

[12], where $d=R_{o}-R_{i}$ is the thickness of the gap between cylinders and $\Lambda$ is the dimensionless curvature of the streamlines and for small gaps $\Lambda=d / R_{i}$ [6].

The definition 4 is not unique, e.g. [8] uses $T a=\frac{R_{i} \omega_{i} d}{\gamma}$, which is in other texts called $R e_{i}$; the second power of definition 4 can be seen as well, e.g. in [11].

A one note to the Görtler problem: the thickness of the ,convective region“ is related to the boundary layer thickness, which varies with the traveled distance ${ }^{4}$ and therefore the Görtler vortices tend to change their size and number density with traveled distance, which makes their observation much more difficult than in the Taylor-Couette problem. This instability is important from the experimentalists point of view as it produces vortices in the confusor of wind tunnel.

When increasing the rotation rate of inner cylinder over the approximate value $T a_{W V F} \approx 46$, the Taylor Vortex Flow becomes wavy (Wavy Vortex Flow). There are suggested two physical mechanisms explaining this transition [6]: the flow inside the Taylor vortex can be seen as a Taylor-Couette system of its own and therefore the same Taylor-Görtler mechanism produces alternating azimuthal motion which is identified as waviness of the vortex tubes. Second possible explanation is based on the

\footnotetext{
${ }^{4}$ it scales as $x^{1 / 2}$
} 
Kelvin-Helmholtz instability, which is often called the basic instability of hydrodynamics [13] and occurs at shear layers, i.e. areas, where the velocity changes. In this case, such role play the boundaries of the streaks ${ }^{5}$. The exact scaling of this transition is not yet available, the answer might be found in the Kelvin-Helmholtz instability of curved free shear layers.

Anyway, the observed [7] wavenumber of this waves varies from 2 at $T a \approx 150$ to 7 when $T a \geq 230$. It can be greater in the case of rotating outer cylinder.

When increasing cylinder speed over $T a_{M W V} \approx 400$, the wavy vortex flow becomes modulated (Modulated Wavy Vortices), i.e. there are more than 1 wave of single wavenumber. One of the important properties of this flow is that the phase between waves on different Taylor vortices is not locked [7].

Some authors distinguish another regime called Chaotic wavy vortex flow, when there is more than 2 waves. Gollub and Swinney [14] defined one possible route to the chaos of an extended dynamical system socalled Ruelle-Takens scenario of transition to turbulence on the basis of Taylor-Couette flow behaviour. Note that even at this stage, the flow is still laminar [11].

On higher speeds, the Taylor vortices lost their laminarity and become turbulent (apparent mainly in the core [15]). Interesting is, that, first, the turbulent vortices are wavy forming the Wavy Turbulent Vortices at $T a_{W T V} \approx$ 650 , and at higher rotation rates, their waviness disappears and the flow is similar to the Taylor vortex flow, but with Turbulent Taylor Vortices. At even higher speeds $(T a>1000)$ the structure of the length-scale of the gap between cylinders disappears in favor of smaller chaotic structures. This stage is called Featureless Turbulence.

The variety of flow regimes becomes much richer, when we rotate also the outer cylinder. Figure 4 shows the map of Taylor-Couette flow regimes as observed by Andereck, Liu and Swinney and published in their breaking work [7].

\subsection{Our planed demonstration apparatus}

We hope that all just described amazing aspects of TaylorCouette flow are able to engage student's interest when they will see all this in vivo together with adequate explanation, what they see. Together with the closeness of this flow and the quite simple geometry, we found this flow to be the ideal one to be prepared as a demonstration apparatus. At this moment (the deadline of the conference proceedings, September 9, 2018) the apparatus is not yet physically realized, we have prepared the instructions for the $3 \mathrm{D}$ printer. The CAD model is shown in figure 5 .

The inner and outer radii in the model are $R_{i}=16 \mathrm{~mm}$ and $R_{o}=20 \mathrm{~mm}$ respectively, the height of the flow section is $h=124 \mathrm{~mm}$ giving the aspect ratio $\Gamma=\frac{h}{d}=35.5$. The rotation rate for achieving the Taylor vortex flow will be $\omega_{T W F}=1.3 \mathrm{rad} \cdot \mathrm{s}^{-1}=12.4 \mathrm{~min}^{-1}$, for wavy vortex flow $\omega_{W V F}=1.5 \mathrm{rad} \cdot \mathrm{s}^{-1}=14 \mathrm{~min}^{-1}$, but the regime of modulated wavy vortices at $\omega_{M W V}=12.5 \mathrm{rad} \cdot \mathrm{s}^{-1}=119 \mathrm{~min}^{-1}$ will be inaccessible.

\footnotetext{
${ }^{5}$ Strong flow between 2 oppositely oriented Taylor vortices.
}

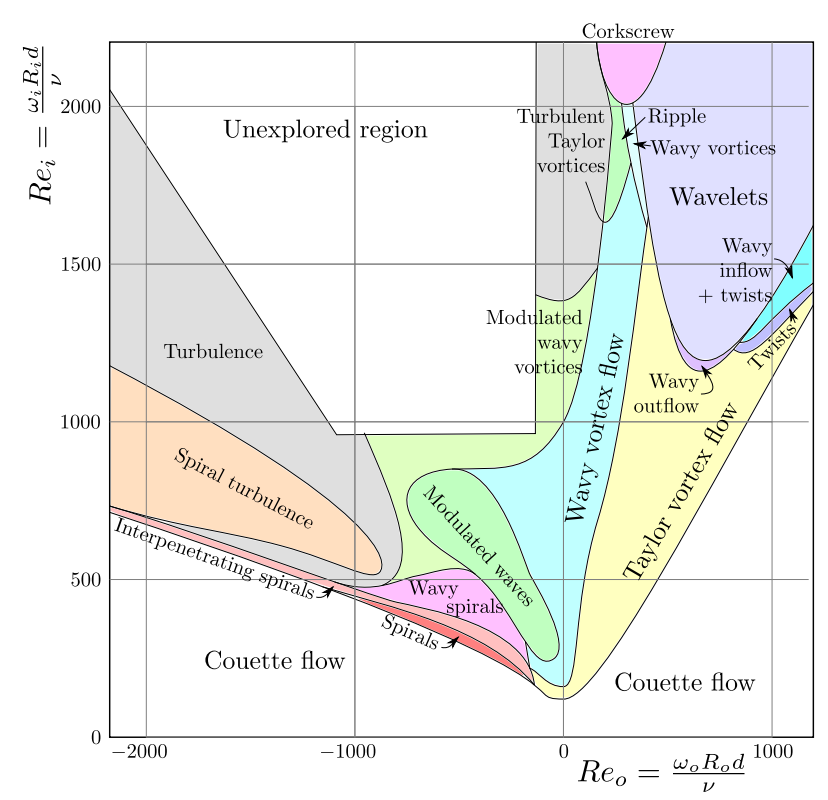

Fig. 4. The map of flow regimes of Taylor-Couette flow between two concentric cylinders as carefully observed and distinguished by Andereck et al. [7]. The parameters are the Reynolds numbers of inner and outer cylinder respectively, $R e_{i, o}=\frac{\omega_{i, o} R_{i, o} d}{v}$, where $\omega_{i, o}$ is the angular velocity, $R_{i, o}$ the radius of inner and outer cylinder respectively and $d=R_{o}-R_{i}$ is the gap between them. The map is adapted from [7].

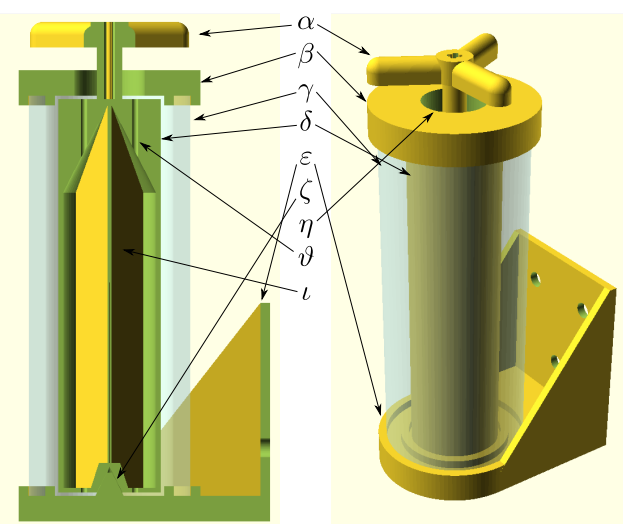

Fig. 5. The CAD model of the planned apparatus drown in program OpenSCAD. $\alpha$ - handle, $\beta$ - top cover, $\gamma-$ outer cylinder made of plexiglass, inner radius $R_{o}=20 \mathrm{~mm}$, thickness $5 \mathrm{~mm}, \delta$ - inner cylinder, $R_{i}=16 \mathrm{~mm}, \varepsilon$ - bottom base, $\zeta$ - cone stick for axial stabilization of the inner cylinder, $\eta$ - hole for the bearing, $\vartheta$ - holes connecting inside of the inner cylinder with the bath, $\iota$ - divider to suppress circulation inside the inner cylinder.

\section{Conclusions}

Taylor-Couette flow is enormously rich in regimes and their transitions, whose order is not yet known, there is lot of triple points as well. The physical mechanism of the first transition from laminar Couette flow to Taylor vortex flow seems to be qualitatively similar to the formation of Bénard cells in thermal convection, the waviness of the Taylor vortices is related to the Kelvin-Helmholtz instability and the turbulent regimes shows all aspects of turbu- 
lent flows - contains all length-scales, its spectrum follow Kolmogorov scaling [16], although it is not homogenous, neither isotropic.

Hence we hope that this flow in connection with the esthetic value of rheoscopic flow visualization technique might attract the attention of students, who would study hydrodynamics due to its amazing nature, not only because it is hard and compulsory curse.

Acknowledgements. D. D. acknowledges the support of MS ZČU program Postdoc 2018, D. D. and M. K. have been supported by the ZČU project Propadovost. We thank to Bohumil Laštovka for valuable technical help and to Radka Lanči for help with project paperwork.

\section{References}

[1] V. Zamarovský, Na počátku byl Sumer (Perfekt, 2004)

[2] L. Skrbek, K.R. Sreenivasan, Physics of Fluids 24 (2012)

[3] I. Zurbenko, The spectral analysis of time series (North-Holland, 1986)

[4] M. Wilkinson, V. Bezuglyy, B. Mehlig, Journal of Fluid Mechanics 667, 158 (2011), 0910. 5330
[5] D.R. Carlson, S.E. Widnall, M.F. Peeters, Journal of Fluid Mechanics 121, 487 (1982)

[6] M.A. Fardin, C. Perge, N. Taberlet, Soft Matter 10, 3523 (2014)

[7] C.D. Andereck, S.S. Liu, S. H.L., J. Fluid Mechanics 164, 155 (1986)

[8] O. Richter, H. Hoffmann, B. Kraushaar-Czarnetzki, Chemical Engineering Science 63, 3504 (2008)

[9] E. Berrich, F. Aloui, J. Legrand, American Society of Mechanical Engineers, Fluids Engineering Division (Publication) FEDSM 1, 1 (2012)

[10] W.S. Saric, Annual Review of Fluid Mechanics 26, 379 (1994), arXiv: 1011.1669v3

[11] V. Uruba, Turbulence (Nakladatelství ČVUT, 2009)

[12] F. Wendt, Ingenieur-Archiv 4, 577 (1933)

[13] A.M. Fridman, Uspekhi Fizicheskih Nauk 178, 225 (2008)

[14] J.P. Gollub, H.L. Swinney, Physical Review Letters 35, 927 (1975)

[15] E. Amromin, Physics of Fluids 19, 13 (2007)

[16] A.N. Kolmogorov, Proceedings of the Royal Society A: Mathematical, Physical and Engineering Sciences 434, 15 (1991) 